\title{
Output Power and SNR Swings in Cascades of EDFA's for Circuit- and Packet-Switched Optical Networks
}

\author{
L. Tančevski, Alberto Bononi, and Leslie A. Rusch, Member, IEEE
}

\begin{abstract}
A simple dynamic model of the erbium-doped fiber amplifier (EDFA) that includes self-saturation by amplified spontaneous emission (ASE) is used to analyze the power and signalto-noise ratio (SNR) transients in wavelength division multiplexed (WDM) optical networks in which signals cross chains of EDFA's from source to destination. The model, which consists of solving sequentially one ordinary differential equation per amplifier, is used to 1) determine power and SNR excursions in the surviving channels along a chain of 35 EDFA's during isolated add-drop events in a 16-channel WDM circuit switching scenario and 2) run Monte Carlo simulations of the first five EDFA's of the same chain fed by burst-mode packet switching traffic on each of the 16 channels. Each packet source is modeled as an ON-OFF asynchronous transfer mode (ATM) source, with ON and OFF times having a heavy-tailed Pareto distribution. The aggregate source model is asymptotically self-similar, and well describes multimedia packet communications. The results are used to examine the influence of average network utilization and source ON-OFF time variance on the probability density function of signal power and SNR at each EDFA output. We demonstrate that selfsimilar traffic generates sizable power and SNR swings, especially at low network utilization. The simulations also indicate sizable broadening of the power and SNR density functions along the cascade of EDFA's, reaching levels in excess of $9 \mathrm{dBm}$ and $4 \mathrm{~dB}$ for the power and SNR swings, respectively, at the 5th EDFA. The effect becomes more pronounced for longer EDFA chains. Such a large broadening may imply serious system impairments in burst-mode WDM packet networks.
\end{abstract}

Index Terms-Asynchronous transfer mode (ATM), communication system traffic, optical communication, optical fiber amplifiers, optical fiber communication, Pareto distributions.

\section{INTRODUCTION}

$\mathbf{E}$ RBIUM-DOPED fiber amplifiers (EDFA's), featuring high gain over a large wavelength range, low intrinsic losses and long fluorescence times, have emerged as a key enabling component in optical systems and networks. In particular, owing to their long fluorescence time, EDFA's in high-speed wavelength division multiplexing (WDM) point to point links do not suffer from the effect of cross-gain saturation, a serious drawback with semiconductor optical

Manuscript received June 18, 1998.

L. Tančevski is with Alcatel Network Systems, Richardson, TX 75081 USA.

A. Bononi is with the Dipartimento di Ingegneria dell'Informazione, Università di Parma, Parma I-43100 Italy.

L. A. Rusch is with the Département de geniéelectrique et de genie informatique, Université Laval, Ste-Foy, P.Q. G1K 7P4 Canada.

Publisher Item Identifier S 0733-8724(99)03814-1. amplifiers. Recently, however, there has been growing interest in estimating the transient time response of EDFA's to gain or loss of one or several wavelength channels in WDM circuitswitching scenario [1]-[6]. In particular, sizeable system impairements may result from channel addition or loss in a point-to-point communication link composed of a long chain of EDFA's. Detailed simulations and experiments have established that the power excursions of the surviving channels due to the power transition on other channels could total several $\mathrm{dB}$, and the speed of the output power transients increases along the chain [2]. Increased output power levels could lead to error bursts, if the power exceeds the threshold for optical nonlinearities; low power levels could lead to inadequate eye opening; ultimately, the receiver's acceptable dynamic range could be exceeded as a combination of these two effects.

In WDM packet-switching burst-mode networks, where no power is transmitted on empty slots, such as nativemode asynchronous transfer mode (ATM), or as the recently proposed WDM internet protocol (WDM-IP) with transport directly onto the optical layer [7], fluctuations of the input powers are present due to the random nature of the packet arrivals at the EDFA, giving rise to a cross-gain saturation effect similar to the one induced by add-drops in circuit switching. Cross-gain saturation in packet networks, however, has not been quantified to date. Network services such as multimedia, variable bit rate (VBR) video applications, and gigabit Ethernet local area networks (LAN) exhibit highly variable traffic characteristics. New studies indicate much increased variability in the packet interarrival times [8], [9] and actual traffic measurements indicate self-similarity in these networks [9]. The significance of the increased variability, leading to self-similarity, from the gain saturation perspective is that longer intervals of higher or lower input power are much more probable, giving enough time to the EDFA to acquire substantially different gain values.

In this paper we investigate the system impairment in such WDM packet networks due to the gain saturation in cascades of EDFA's fed by input traffic with various degrees of variability in the packet interarrival times. Through numerical simulations, we demonstrate sizable power and optical signalto-noise ratio (SNR) swings at the output of the EDFA's.

The organization of the paper is as follows. In Section II, we describe the EDFA model used to calculate the transient 


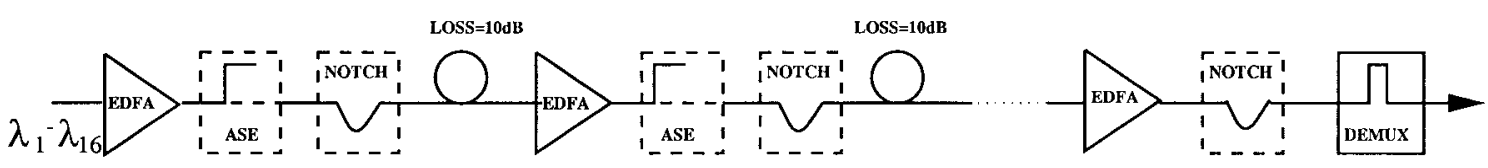

Fig. 1. Diagram of the simulated cascade of EDFA's.

responses of EDFA's. It is based on the model introduced in [1] with the amplified spontaneous emission (ASE) being added as outlined in [11] and [12]. In Section III, we give both power and SNR transients along an EDFA chain caused by a single power discontinuity, such as an add-drop in a circuit-switching scenario. We evaluate the speed of the transients through the figure of merit $1-\mathrm{dB}$ time, that is, the time during which the power excursions reach the 1 $\mathrm{dB}$ level. We derive simple expressions for the evolution of the 1-dB times along a cascade of EDFA's. We present the results of a simulation of a circuit-switched communication link comprising 16 WDM channels transmitted through a cascade of 35 EDFA's. Simulations address the steady-state SNR dependence on wavelength, the 1-dB times of power and SNR transients and the power and SNR excursions of eight of the 16 channels. The excursion results are used in the subsequent sections to describe the simulations of the packetized traffic.

In Section IV, we present a brief description of the packet traffic model employed to simulate packet traffic with various degrees of variability. The model is based on the superposition of many ON-OFF periods each having a Pareto heavy-tailed distribution. Relevant definitions are given for the traffic parameters employed.

In Section V, we present results for the same cascade as before, fed in this case by packetized traffic with various degrees of variability. The simulations are run over one million transmitted slots. We present histograms estimating the probability density function of the power and SNR swings at the output of the first and of the fifth EDFA. The results are compared for various degrees of variability, ranging from relatively smooth traffic to self-similar traffic, and for various network utilization factors. We demonstrate that the swings are much more pronounced further along the cascade, stemming from transients that become progressively faster along the cascade. Also, the SNR swings are found to be smaller than the corresponding power swings due to the compensating effect of transients in the ASE itself. It is argued that highly variable traffic (such as self-similar traffic) coupled with low utilization, could lead to a serious deterioration of system performance. Power swings in excess of $9 \mathrm{dBm}$ and SNR swings in excess of $4 \mathrm{~dB}$ are observed during the simulation and indications are that the effect will be much more pronounced in longer cascades. Finally, we draw some brief conclusions.

\section{EDFA MODEL}

The dynamic behavior of the EDFA is obtained by the model introduced in [1] and based on solving a single ordinary differential equation (ODE), into which ASE is included as outlined in [11], [12]

$$
\begin{aligned}
P_{k}^{I S}[\tau & \left.\frac{d}{d t} G_{k}(t)+G_{k}(t)+A_{k}\right] \\
= & -\sum_{j=0}^{N} P_{j}^{i n}(t)\left\{e^{\left[\left(G_{k}(t)+A_{k}\right) P_{k}^{I S} / P_{j}^{I S}-A_{j}\right]}-1\right\} \\
& -P_{\mathrm{ASE}}(t)
\end{aligned}
$$

Here $N$ is the number of WDM channels, channel 0 indicating the pump; $\tau$ is the EDFA fluorescence time $P_{k}^{\text {in }}, P_{k}^{\text {out }}$, and $P_{k}^{I S}$ are the input, output, and intrinsic saturation fluxes (photons/s) at wavelength $\lambda_{k} ; G_{k} \triangleq \ln \left(P_{k}^{\text {out }} / P_{k}^{\text {in }}\right)$ is the logarithmic gain $A_{k}=\alpha_{k} L$ is the loss parameter, $\alpha_{k}$ being the absorption constant at $\lambda_{k}$ and $L$ being the length of the amplifier; $P_{\mathrm{ASE}}(t)$ is the amplifier (statistical) average spontaneous emission flux (photons/s) calculated assuming a constant inversion as [12], [13]:

$$
P_{\mathrm{ASE}}(t)=4 \int\left(e^{G(\nu, t)}-1\right)\left[\frac{\sigma^{e}(\nu)}{\sigma^{T}(\nu)} \frac{(G(\nu, t)+A(\nu))}{G(\nu, t)}\right] d \nu
$$

where $\sigma^{e}(\nu)$ and $\sigma^{a}(\nu)$ are the emission and absorption crosssections, $\sigma^{T}(\nu) \triangleq \sigma^{a}(\nu)+\sigma^{e}(\nu), G(\nu)$ is the log-gain and $A(\nu)$ the loss, all at frequency $\nu$. In (2), the term in square brackets represents the spontaneous emission factor $n_{\mathrm{sp}}$, and the factor four accounts for two different polarizations and two different directions of propagation of the ASE; when calculating the output SNR, only half of that value represents the forward ASE.

\section{TRANSIENTS CAUSED BY A Single POWER DisCONTINUITY}

The EDFA chain in analysis is adopted from [14] and shown in Fig. 1. It is composed of 35 identical EDFA's, with absorption and emission cross-sections, saturation powers, and absorption coefficients as in [6]. Forward pumping at $980 \mathrm{~nm}$ with pump power of $18.4 \mathrm{dBm}$ and EDFA length $14.0 \mathrm{~m}$ are assumed. The chain is designed for a 16-channel WDM (circuit-switching) system in the wavelength band 1544-1559 $\mathrm{nm}$, with $1 \mathrm{~nm}$ spacing and $-7 \mathrm{dBm}$ input power. With this specific EDFA configuration and input fluxes, the gain peak is around $1530 \mathrm{~nm}$ and thus a passband filter after every amplifier is inserted to block the propagation of the forward ASE below $1540 \mathrm{~nm}$, which would otherwise saturate the chain [14]. A notch-filter for gain equalization is also placed after every fourth EDFA. A Lorentzian shape for its attenuation 


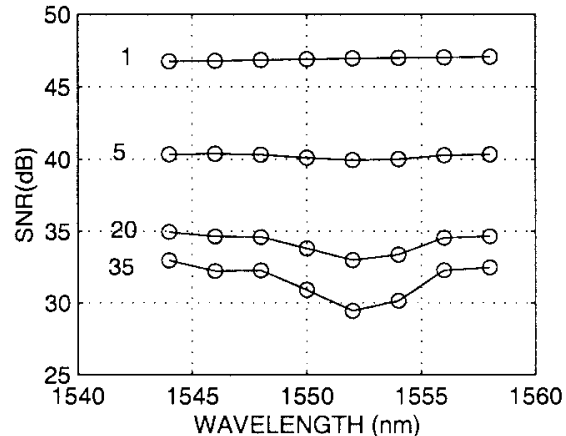

(a)

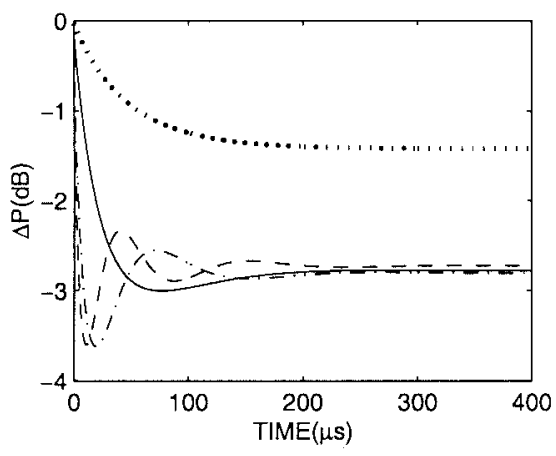

(c)

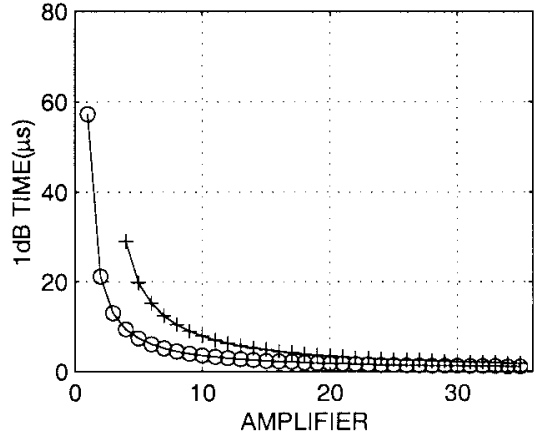

(b)

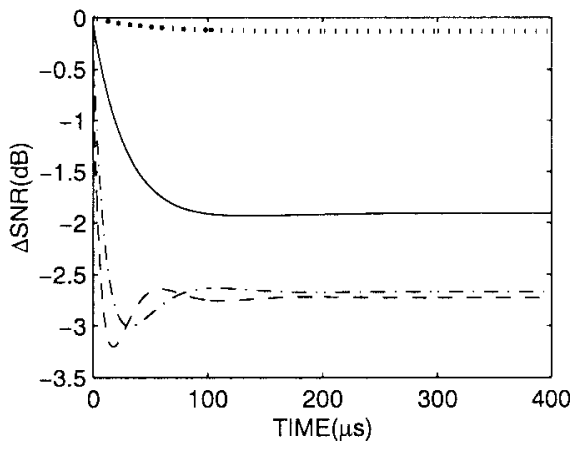

(d)

Fig. 2. Simulation of the point-to-point link: (a) steady-state optical SNR at the output of the first, fifth, twentieth, and the thirty-fifth EDFA, (b) 1 dB times for power excursion (circles) and for SNR excursion (crosses), (c) Power excursion, and (d) SNR excursion. dotted line-first EDFA, solid line-fifth EDFA, dashed-dotted line-twentieth EDFA, dashed line-thirty-fifth EDFA.

characteristic is assumed [14], [15]:

$$
A_{\text {notch }}(\lambda)=1-\frac{1-A_{0}}{1+\left[\frac{\lambda-\lambda_{c}}{\Delta \lambda}\right]^{2}}
$$

with filter depth $A_{0}=1.4 \mathrm{~dB}$, center wavelength $\lambda_{c}=1546$ $\mathrm{nm}$, and full-width at half-maximum (FWHM) $\Delta \lambda=2.5 \mathrm{~nm}$. The interamplifier loss is $10 \mathrm{~dB}$. Before the receiver, an optical demultiplexer is inserted to filter out the desired signal, the width of the optical filter being $0.125 \mathrm{~nm}$.

We next show the transients along the chain caused by an isolated discontinuity of the input power. At time $t=$ 0 , the system is at steady state and only eight of the 16 channels (the odd channels) are present. The steady state is calculated by sequentially setting $d G / d t=0$ in (1) for each EDFA and solving the resulting transcendental equation. For ASE computations, the integral in (2) is calculated over 64 contiguous frequency bands, between 1518 and $1581 \mathrm{~nm}$, with $1 \mathrm{~nm}$ separation. With the above input powers the cascade is balanced, in the sense that each EDFA can provide roughly $10 \mathrm{~dB}$ gain to the eight active channels, to counter the interamplifier losses. Fig. 2(a) shows the optical SNR of the active odd channels at the first, fifth, twentieth, and thirty-fifth EDFA outputs. Circles help identify the channels. Note that the system features a fairly flat gain profile, as the SNR spread is less than $3 \mathrm{~dB}$, in good agreement with the results in [14], [16].

At time $t=0^{+}$, i.e., one instant after time zero, the remaining eight even channels are reinstated and the transients are determined by sequentially solving the ODE (1) for each
EDFA using a Runge-Kutta routine of the fourth order, in $0.1 \mu$ s time increments. The associated $1-\mathrm{dB}$ times for power and SNR excursions versus amplifier number in the cascade are shown in Fig. 2(b) for the channel at $1552 \mathrm{~nm}$, the one at the center of the notch. We calculate the optical SNR as the ratio of the signal power to the ASE statistical average power (2) over a bandwidth of $0.125 \mathrm{~nm}$ centered at the signal wavelength. Circles and crosses show the theoretical values obtained as detailed in the Appendix. We see that transients become faster along the cascade [2]. Note that the transients of the output power are faster than the transients of the optical SNR. Fig. 2(c) and (d) shows the power and SNR $\mathrm{dB}$-excursion for the channel at $1552 \mathrm{~nm}$ at the output of the 1st (dotted line), 5th (solid line), 20th (dash-dotted line) and the 35th EDFA (dashed line). Note that toward the beginning of the cascade the SNR excursion is significantly smaller than the power excursion, but further along the cascade the power and SNR excursions become comparable. In fact, as is also evident from Fig 2(b), the SNR excursion at the output of the first, the second and the third EDFA in the cascade never reaches the $1 \mathrm{~dB}$ level and only at the output of the fourth EDFA does it cross the $1 \mathrm{~dB}$ mark. The results of Fig. 2, albeit obtained for an add transient in a circuit switching scenario, will be used in the next sections to interpret the results of the simulation for packetized traffic.

\section{Packet TRAFFiC Model}

Fast fluctuations of the input power are inherently present when dealing with packetized data in WDM burst-mode op- 


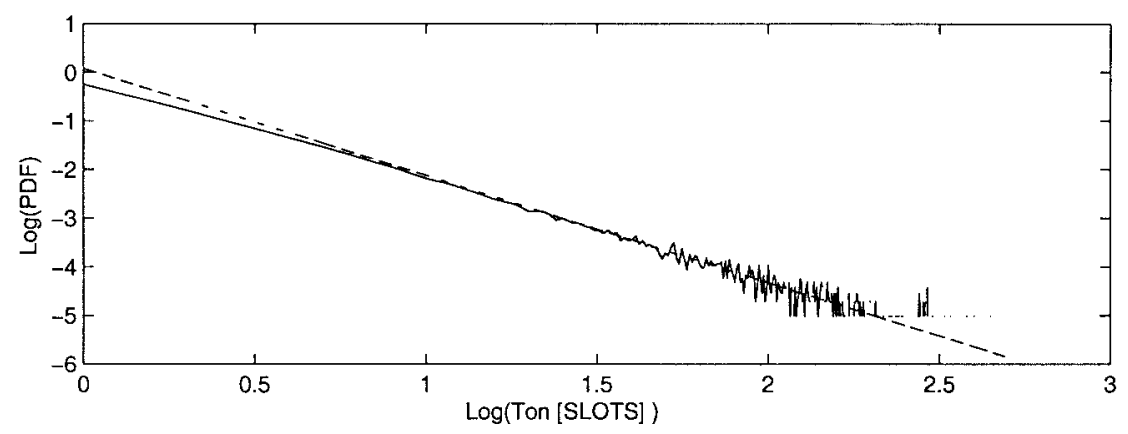

(a)

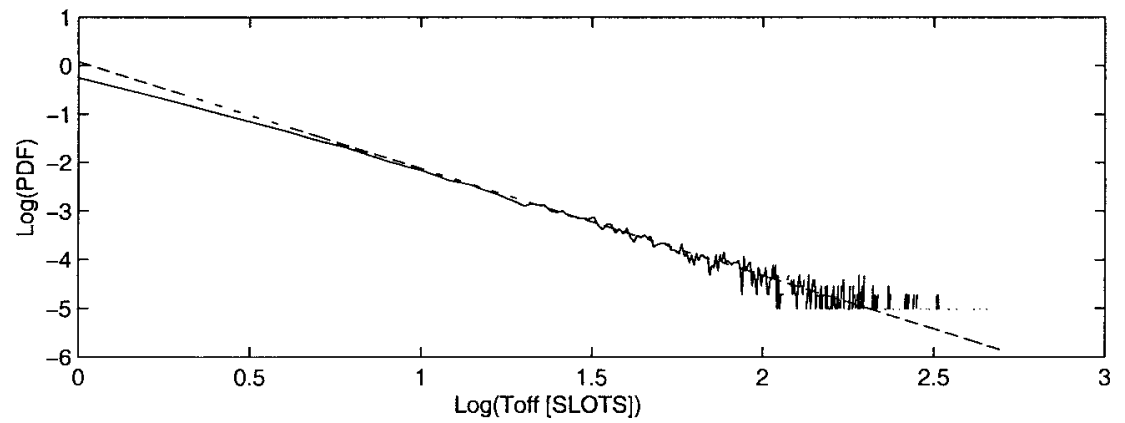

(b)

Fig. 3. Log-Log histogram of the normalized ON-OFF periods with Pareto distribution for $\alpha_{\text {off }}=\alpha_{\text {on }}=1.2$ and $\rho=0.5$ : (a) histogram of the ON periods and (b) histogram of the ofF periods. Full line—simulation of the rounded Pareto distribution; dashed line—analytical nontruncated Pareto distribution.

tical networks. Recently it was discovered that Ethernet LAN traffic [17], [8], [18], or VBR video traffic over ATM [9] exhibit long-range dependence and self-similarity. The role of self-similarity or indeed high variability in interarrival times from the EDFA gain saturation perspective is that long periods of less traffic (OFF periods) or higher traffic (ON periods) are far more likely, giving enough time to the EDFA to respond to the fluctuations of the input power. The resultant fluctuations in gain, output power, and SNR leads to deterioration of system performance.

The traffic in our simulations consists of 16 independent sources, one for each wavelength channel. Each of these sources is modeled as a succession of oN periods (intervals during which packet bursts are transmitted) and OFF periods (intervals during which no packet is transmitted). The duration of each $\mathrm{ON}-\mathrm{OFF}$ period is assumed to be a random variable $T_{i}, i \in\{$ on, off $\}$, with Pareto distribution, having cumulative distribution function [19]

$$
F_{i}(t)=1-\left(\frac{t_{0}}{t}\right)^{\alpha_{i}}
$$

with finite mean $E\left[T_{i}\right]=\left(t_{0} \alpha_{i} / \alpha_{i}-1\right)$ when $\alpha_{i}>1$. In [17] it was proven that the superposition of infinitely many ON-OFF sources, each having infinite variance, produces self-similar traffic. In particular, with a Pareto distribution, when $\alpha_{i}<2$ the variance is infinite, while when $\alpha_{i}>2$ the variance is

$$
\operatorname{VAR}\left[T_{i}\right]=t_{0}^{2} \frac{\alpha_{i}}{\left(\alpha_{i}-1\right)^{2}\left(\alpha_{i}-2\right)}
$$

We will use the $\alpha_{i}$ parameters as indicators of the degree of variability of the traffic. In the simulations we used $t_{0}=1$, so that the packet burst duration (ON time) and packet burst spacing (OFF time) can vary from 1 to $\infty$ slots. Given a random variable $U$ with uniform distribution on $[0,1]$, we use the following transformation to generate a random variable $P$ with a truncated Pareto distribution

$$
P=\left\lfloor\frac{t_{0}}{U^{1 / \alpha}}\right\rfloor
$$

where rounding to the smallest integer $(\lfloor\rfloor)$ is necessary in a slotted system such as ATM so that the duration of the ON and OFF periods is a multiple of the packet size.

The overall network utilization is given as $\rho=$ $\left(E\left[T_{\text {on }}\right] /\left(E\left[T_{\text {on }}\right]+E\left[T_{\text {off }}\right]\right)\right)$. Given the utilization $\rho$ and the mean of the oN periods $E\left[T_{\text {on }}\right]$, the $\alpha_{\text {off }}$ parameter is obtained as

$$
\alpha_{\mathrm{off}}=\frac{(1-\rho) \alpha_{\mathrm{on}}}{(1-\rho) \alpha_{\mathrm{on}}-\rho\left(\alpha_{\mathrm{on}}-1\right)} .
$$

Histograms of the simulated ON and OFF periods (expressed in slots) are shown in Fig. 3 in solid line on a log-log scale. They are obtained by simulating one million slots, with $\rho=0.5, \alpha_{\text {on }}=\alpha_{\text {off }}=1.2$ (infinite variance). The sample mean is 4.9 slots and the histograms go over 102938 counts each. The histograms are estimators of the probability density function (pdf) of the truncated Pareto random variables $T_{\text {on }}$ and $T_{\text {off }}$. For comparison, the pdf of a (nontruncated) Pareto distribution, calculated using (4), is also given in dashed line. Note that the logarithm of the Pareto pdf has slope $-(1+\alpha)$, as is evident in the figures. The discrepancy between the simulated and the analytical curve in the region of small ON-OFF values is due to truncation. Note that the monitoring time was not sufficiently long to observe the heaviest tails of the distribution; the longest observed duration was around 500 
slots. The finite monitoring time also skews the sample mean of the truncated Pareto distribution: 4.9 slots versus six slots for the theoretical mean of a standard Pareto distribution.

\section{Transients Caused by Burst-Mode Packet TrafFic}

We present in this section the results of numerical simulations performed on the chain described in Section III, when fed by the packetized traffic described above. Our goal is to quantify the impact of the gain dynamics caused by rapid fluctuations of the input traffic on the output power and optical SNR. Slotted, native-mode ATM, in which the slots from all 16 channels arrive aligned at each EDFA and packets (called cells) are of fixed size of 53 bytes, is assumed throughout, although the simulation results apply also to a WDM IP transport, with IP packets of variable length but still an integer multiple of some cell length. Unless otherwise stated, the following holds: the bit rate is $150 \mathrm{Mb} / \mathrm{s}$, corresponding to a cell duration of $2.82 \mu \mathrm{s}$; the ODE (1) was solved in ten points per cell, resulting in a time resolution of $0.3 \mu \mathrm{s}$. Note that $0.3 \mu \mathrm{s}$ is well below the $7.4 \mu \mathrm{s} 1-\mathrm{dB}$ time for the fifth EDFA indicated in Fig. 2(b) and hence the time resolution is fine enough to capture power and SNR transients much less than $0.1 \mathrm{~dB}$. To effectively simulate the highly variable input traffic, over one million slots were transmitted, corresponding to $3 \mathrm{~s}$ of transmission. All the relevant amplifier parameters are the same as in the case of the cascade of 35 EDFA's described previously. The peak input cell power/ch is $P_{p}=-7 \mathrm{dBm}$.

Due to the long run times required, the first five EDFA's only were simulated. The power (and optical SNR) were monitored at the output of the first and of the fifth EDFA for the wavelength channel at $\lambda=1552 \mathrm{~nm}$. The output powers (SNR's) on slots carrying a cell were calculated in ten points per slot and arranged into a histogram calculated on 100 bins of width $0.2 \mathrm{~dB}$, uniformly tiling the range between $\bar{X}-10$ and $\bar{X}+10 \mathrm{dBm}(\mathrm{dB})$, where $\bar{X}$ is the steady-state value of the power (SNR) for the given utilization $\rho$, obtained from (1) by setting $d G / d t=0$, and using all 16 active (i.e., continuously ON) channels, each with input flux $\rho P_{p}$ at the first EDFA. Obviously at $\rho=0.5$ the "average" gain in the chain is the same as that of the balanced chain of Section III with the eight even channels present, with a gain of roughly $10 \mathrm{~dB}$ balancing the interamplifier losses.

In order to keep the system well balanced on average for different utilizations, i.e., to keep the same value of $\bar{X}$, the pump power was changed for different utilizations according to (12). If the utilization changes from $\rho$ to $\rho^{\prime}$, the input flux of channel $j$ at amplifier $i$ changes from $P_{i, j}$ to $P_{i, j}^{\prime}=\left(\rho^{\prime} / \rho\right) P_{i, j}$, so that we use $\Delta P_{i, j}=\left(\left(\rho^{\prime} / \rho\right)-1\right) P_{i, j}$ in (12) to find the required pump variation. Specifically, the pump power at the first EDFA was $18.4 \mathrm{dBm}$ for utilization $\rho=0.5,20.0 \mathrm{dBm}$ for utilization $\rho=0.7$ and $16.4 \mathrm{dBm}$ for utilization $\rho=0.3$.

In analyzing the results of the simulation, it is convenient to define several parameters in the histogram representation. A sketch of a histogram of the simulated pdf of the output power is shown in Fig. 4. Results for slowly varying traffic are shown with a solid line. This curve is nearly vertical and centered around the steady state (indeed the most probable) value $\bar{X}$.

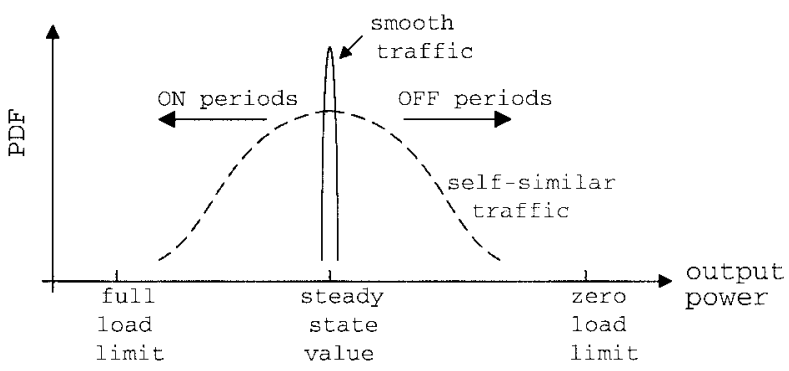

Fig. 4. The histogram plot with its relevant parameters.

As the variability of the ON and OFF periods increases (that is to say, the variance of these periods increases) the curve broadens and assumes the shape shown with dashed line in Fig. 4. We note two limit points in Fig. 4, dubbed the full load limit and the zero load limit. The full load limit occurs when all the packets at all the wavelengths are present for a long period of time, causing the EDFA to saturate to the limit and to assume small values for the gain (this, in effect, corresponds to utilization $\rho=1$ ), hence, the output power is at its minimum. The zero load limit occurs when long periods of empty slots at all wavelengths (lulls) are present, giving time to the EDFA gain to recover its small signal value, thus causing high-power levels and gain sag across the cells following the lulls. This zero load limit is usually far to the right, as the gain and the corresponding output power are very large. The histogram cannot exceed either of these two limits. Variability or high variance in the OFF periods will cause broadening of the curve toward the zero load limit; likewise, variability in the ON periods will broaden the curve toward the full load limit. The degree of broadening depends on the variability of the ON-OFF periods but also depends on the speed of the transients as will be established later.

We begin in Fig. 5 with an analysis of the output power fluctuations at the first amplifier for the wavelength channel at $\lambda=1552 \mathrm{~nm}$. SNR excursions are not considered for the first amplifier, as results presented in Fig. 2(d) show they are not significant this early in the amplifier chain. We consider first in Fig. 5(a) a utilization factor of $\rho=0.5$; the steady-state power is $\bar{X} \cong 3.1 \mathrm{dBm}$, corresponding to a steady-state gain of 10.1 $\mathrm{dB}$. The zero load limit of is about $6 \mathrm{dBm}$, corresponding to a small signal gain of roughly $13 \mathrm{~dB}$. To calculate the full load limit we make use of data for continuous traffic in Fig. 2(c), where the difference between time $t=0$ with eight of 16 channels present $(\rho=0.5)$ and time $t \gg 0$ with all channels present $(\rho=1)$ is seen to be $1.5 \mathrm{~dB}$. Hence, in Fig. 5(a) the full load limit lies around 3.1-1.5 =1.6 dBm. Curve D is the simulated pdf of the output power for relatively smooth input traffic $\left(\alpha_{\mathrm{on}}=\alpha_{\text {off }}=5.0\right)$ and is very compact, i.e., very little fluctuation is visible. Indeed, since the traffic is smooth, the occurrence of long OFF (less input power than the steady-state value) or long ON (more input power than the steady state value) periods is unlikely and hence the output power stays mostly at its steady state value. As the variability of the traffic increases (curve $\mathrm{C}$ representing the case of $\alpha_{\mathrm{off}}=\alpha_{\mathrm{on}}=2.1$ ) the curve broadens, indicating increased likelihood that the output power will vary significantly from the steady-state 


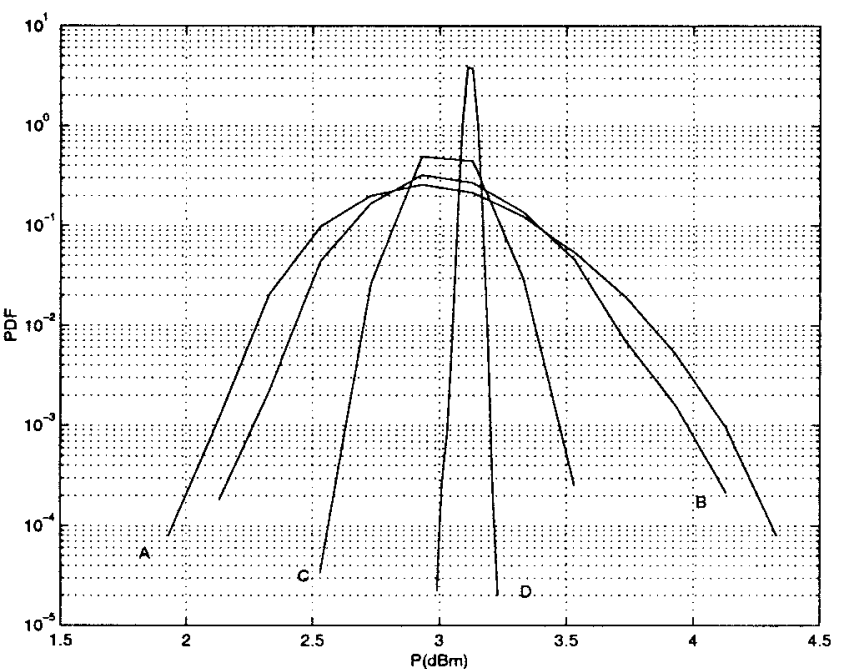

(a)

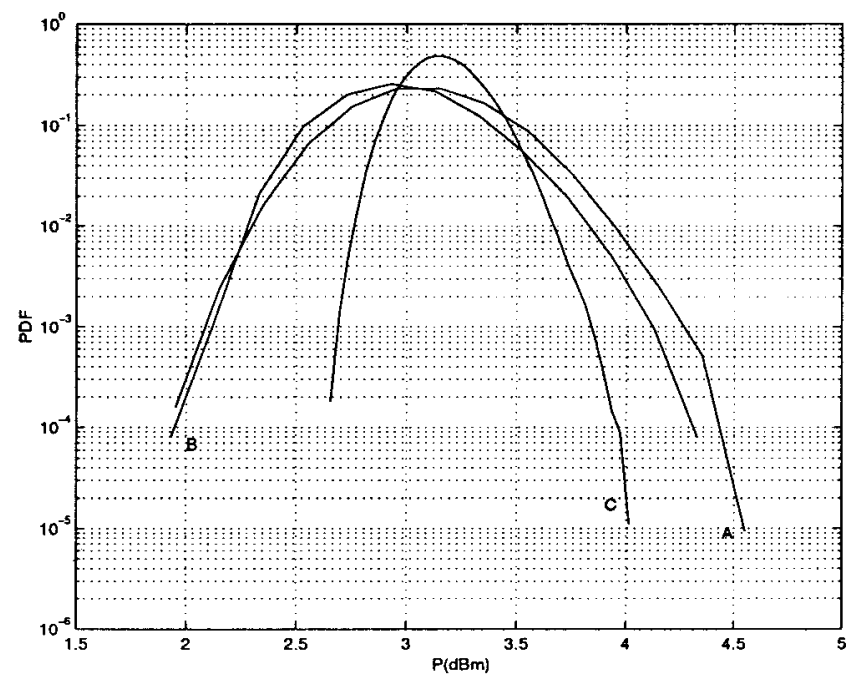

(b)

Fig. 5. Estimated probability density function of the powers: (a) output of the first EDFA: utilization $\rho=0.5$ and various $\alpha$ parameters: $\alpha_{\text {off }}=\alpha_{\text {on }}=1.2$ (curve A); $\alpha_{\text {off }}=\alpha_{\text {on }}=1.2$ and $2.5 \mathrm{~Gb} / \mathrm{s}$ (curve B); $\alpha_{\text {off }}=\alpha_{\text {on }}=2.1$ (curve C); $\alpha_{\text {off }}=\alpha_{\text {on }}=5.0$ (curve D). (b) Self-similar case and various utilizations: $\alpha_{\text {on }}=1.64, \alpha_{\text {off }}=1.2 \rho=0.3$ (curve A); $\alpha_{\text {on }}=1.2$, $\alpha_{\text {off }}=1.2 \rho=0.5$ (curve B); $\alpha_{\text {on }}=1.2, \alpha_{\text {off }}=1.64 \rho=0.7$ (curve C).

value. The self-similar case representing input traffic with infinite variance of ON/OFF times is depicted with curve $\mathrm{A}$ ( $\alpha_{\text {off }}=\alpha_{\text {on }}=1.2$ ) where it is evident that sizable power fluctuations occur, the range of power swings covering almost $2.5 \mathrm{dBm}$ with a probability larger than $10^{-4}$. The broadening of the curve occurs on both sides of the maximum probability level (the steady state level) due to the infinite variance of both OFF and ON periods. Note that curve A comes very close to the full load limit. Curves A, C, and D are all calculated assuming a bit rate of $150 \mathrm{Mb} / \mathrm{s}$. To investigate the influence of the bit rate on the power swings, simulation of the selfsimilar case with $\rho=0.5, \alpha_{\text {off }}=\alpha_{\text {on }}=1.2$ and bit rate of $2.5 \mathrm{~Gb} / \mathrm{s}$ was also performed and the results are depicted in Fig. 5(a), curve labeled B. Since the packet duration is only $0.17 \mu \mathrm{s}$, the transmission of 5 million slots was monitored, while the transients were calculated only in two points per slot, again giving a total of ten million points, as in the previous simulations (one million slots and ten points per slot). It is evident that the broadening, as compared to curve A, is less pronounced in both directions of the maximum level. This is hardly a surprising result, as in the case of $2.5 \mathrm{~Gb} / \mathrm{s}$ the absolute duration of the ON or OFF periods is shorter (due to the shorter durations of the slots) and hence the EDFA has less time to assume different gain values. There is nonetheless a substantial broadening of the output power pdf, even at this high bit rate. Another point to note on Fig. 5(a) is the offset of the maximum levels of the curves; we would expect the maximum of the pdf to occur at the steady state value $\bar{X}$. The maximum level of curve D occurs exactly at the value calculated using the steady-state model in Fig. 2(c), but the subsequent curves $\mathrm{A}, \mathrm{B}$, and $\mathrm{C}$ show an offset which increases toward lower power values as the variability increases and reaches almost $0.5 \mathrm{dBm}$ for curve A. This can be ascribed to the long monitoring times needed to see the effect of the self-similarity. This is supported by the fact that curve B, which represents simulation with exactly the same degree of variability as curve A, displays a smaller offset (in curve B five million slots were transmitted as opposed to one million in curve A, and hence the simulation of the self-similar case was more accurate).

To investigate the effect of the utilization factor $\rho$ on the power swings, we performed self-similar simulations for different values and the results are represented in Fig. 5(b). Curve B stands as a reference curve and is the self-similar case with $\alpha_{\text {off }}=\alpha_{\text {on }}=1.2$ and $\rho=0.5$, that is to say, the same curve as curve A in Fig. 5(a). A smaller utilization factor of $\rho=0.3$ and $\alpha_{\text {off }}=1.2, \alpha_{\text {on }}=1.64$ is depicted with the curve A. The broadening is larger, due to the fact that the variability in OFF periods can contribute to a more dramatic effect, hence the broadening on the right. Note that the full load limit for curve A stands at around $0.6 \mathrm{dBm}$, whereas that for curve $\mathrm{B}$ is around $1.7 \mathrm{dBm}$. The case with $\rho=0.7, \alpha_{\text {off }}=1.64$ and $\alpha_{\text {on }}=1.2$ is depicted in curve C. Note that the broadening at $\rho=0.7$ is substantially smaller, which can be attributed to much shorter OFF periods. The effect of variability is much less pronounced, as evident from the relatively small broadening on the right. The effect of the variability in ON periods, however, is more pronounced because the $\mathrm{ON}$ periods are longer and the power swings already closely approach the full load limit (about $2.53 \mathrm{dBm}$ ).

It should be emphasized that the degree of broadening of the power curves depends very strongly on the speed of the transients: the faster the transients, the larger the broadening. This point can be made stronger by comparing simulations presented here with different simulations reported in [20]. The referenced simulations were undertaken for much higher input powers/ch $(-3 \mathrm{dBm} / \mathrm{ch})$ and for EDFA's with much longer length $(35 \mathrm{~m})$ and very high gain (34 dB small signal gain), designed again to provide roughly $10 \mathrm{~dB}$ gain/ch. All other EDFA and network parameters were the same. Fig. 6(a) represents the self-similar case with $\rho=0.5, \alpha_{\text {off }}=\alpha_{\text {on }}=1.2$ as calculated with $-7 \mathrm{dBm} / \mathrm{ch}$ and EDFA length $14.0 \mathrm{~m}$ [curve A, the same as curve A on Fig. 5(a) and curve B on Fig. 5(b)] and the case of $-3 \mathrm{dBm} / \mathrm{ch}$ and EDFA length of $35.0 \mathrm{~m}$ 


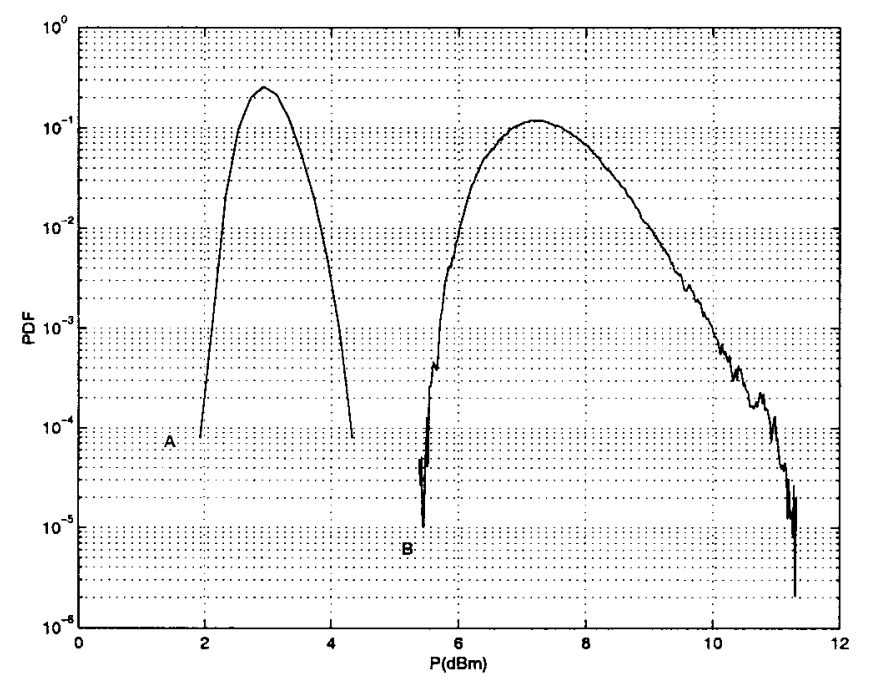

(a)

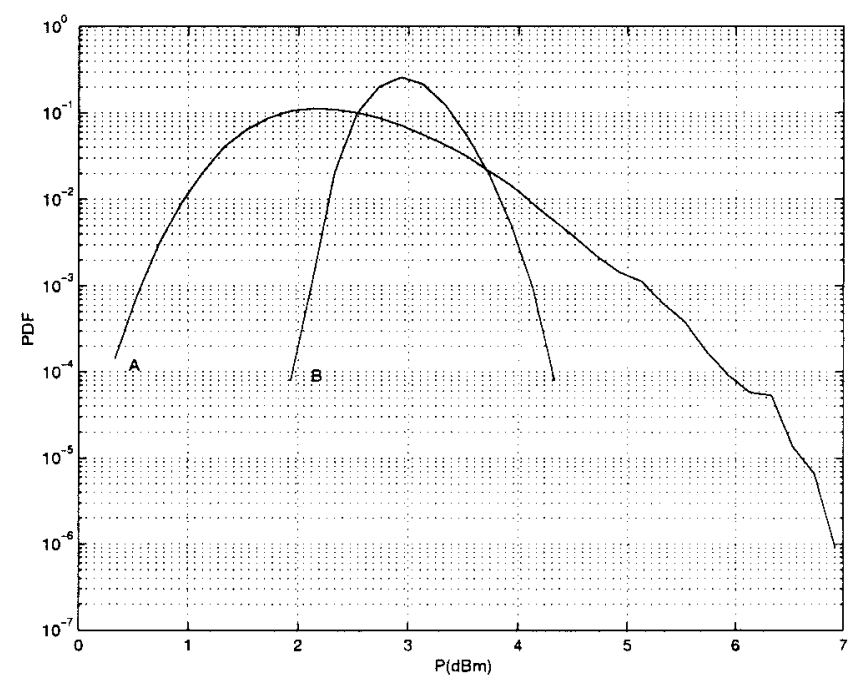

(b)

Fig. 6. Estimated probability density function of output powers: (a) output of the first EDFA for $-7 \mathrm{dBm} / \mathrm{ch}$ and length of the EDFA $14.0 \mathrm{~m}$ (curve A) and $-3 \mathrm{dBm} / \mathrm{ch}$ and length of the EDFA $35.0 \mathrm{~m}$ (curve B) and (b) output of the first EDFA (curve B) and of the fifth EDFA (curve A) for $-7 \mathrm{dBm} / \mathrm{ch}$ and EDFA length $14.0 \mathrm{~m}$. For all curves, $\rho=0.5$ and $\alpha_{\text {off }}=\alpha_{\text {on }}=1.2$.

(curve B). It is evident that the broadening in the case of the curve $\mathrm{B}$ is much larger, the power swings covering a range of almost $5.5 \mathrm{dBm}$ with probability $>10^{-4}$. Recall that the $1-\mathrm{dB}$ time depends on the amount of power change [see (8) and (9)], which, in this case, is substantially larger, resulting in faster transients. Indeed, calculations indicate that the 1$\mathrm{dB}$ time at the output of the first EDFA in the case of -3 $\mathrm{dBm} / \mathrm{ch}$ described in [20] is $15 \mu \mathrm{s}$ as opposed to $57 \mu$ s visible on Fig. 2(b). This establishes that the $1 \mathrm{~dB}$ time has profound influence on the power excursions and the broadening of the curves. Also note that in Fig. 5(a) curves A and B are shifted by $4 \mathrm{dBm}$ relative to each other, consequence of the fact that the input powers are -7 and $-3 \mathrm{dBm} / \mathrm{ch}$, respectively, while the gain in both cases is about $10 \mathrm{~dB}$.

Very similar calculations were done for the case of the output power and optical SNR in a cascade of EDFA's. We examine the histograms of output powers and SNR's at the output of the fifth EDFA in the cascade. Returning to Fig. 2(b), note that the $1 \mathrm{~dB}$ time decreases progressively along the cascade $(1 \mathrm{~dB}$ time at the first EDFA is $57 \mu \mathrm{s}$ and at the fifth EDFA is $7.4 \mu \mathrm{s}$ ), both for the power and SNR excursion, meaning that the transients along the cascade accelerate. The resulting effect of this acceleration is to give a much larger broadening of the curves at the output of the amplifiers further along the cascade. Fig. 6(b) compares the power histograms at the output of the first EDFA (curve A) and the fifth EDFA (curve $\mathrm{B}$ ). While the power swings cover only $2.5 \mathrm{dBm}$ for the case of a single EDFA, that range grows to nearly $7 \mathrm{dBm}$ at the output of the fifth EDFA, a very substantial broadening indeed.

We next present the power and SNR pdf's obtained at the output of the fifth EDFA The analysis of the power swings in the cascade was performed in exactly the same manner as was described for a single EDFA. The influence of the degree of variability of the input traffic on the power swings is represented on Fig. 7(a) for the case of $\rho=0.5$. As pointed out earlier, smoother traffic results in modest broadening of the power curve and curve D ( $\left.\alpha_{\text {off }}=\alpha_{\text {on }}=5.0\right)$ features only $1 \mathrm{~dB}$ broadening. As the traffic variability increases, the broadening increases as well, reaching almost $7 \mathrm{dBm}$ for the self-similar case of $\alpha_{\text {off }}=\alpha_{\text {on }}=1.2$ depicted with curve A. Again, the simulation with $2.5 \mathrm{~Gb} / \mathrm{s}$ bit-rate (curve B) features less broadening than the corresponding one at $150 \mathrm{Mb} / \mathrm{s}$ (curve A). Very similar conclusions as in the case of a single EDFA hold, and will not be repeated. We note, however, that the full load limit in this case [see Fig. 2(c)], lies $2.7 \mathrm{dBm}$ on the left of the maximum probability level, that is, at $0 \mathrm{dBm}$ on Fig. 7(a). The zero load limit, however, can be determined (neglecting the influence of the notch-filter) as follows: $-7+4 \times(13-10)+13$ and lies at approximately $18.0 \mathrm{dBm}$ in Fig. 7(a). We also note that the steady-state power value (the maximum probability level), due to the behavior of the cascade, is shifted by roughly 0.5 $\mathrm{dB}$ relative to the value at the output of the first EDFA, and stands at around $2.7 \mathrm{dBm}$. Fig. 7(b) depicts the self-similar case for different utilization factors and as such is equivalent to Fig. 5(b). Again, it is obvious that the amount of broadening increases when the utilization factor decreases, for the same reasons discussed previously. We note that the full load limits are as follows: at $1.5 \mathrm{dBm}$ for utilization $\rho=0.7$ (curve $\mathrm{C}$ ); at $0 \mathrm{dBm}$ for utilization $\rho=0.5$ (curve B) and; at -2.1 $\mathrm{dBm}$ for $\rho=0.3$ (curve A). In this respect, curve $\mathrm{C}$ already reaches that limit and further broadening in that direction is not possible.

The histograms of the output optical SNR's are presented in Fig. 7(c) and (d). Note that the maximum probability levels (the steady-state levels) coincide with those represented in Fig. 2(a). Also note that the broadening of the SNR curves is much smaller than that of the power curves. This is due to the fact that the transients of the SNR are much slower, as evident also from Fig. 2(b) which represents the $1 \mathrm{~dB}$ times. In fact, the $1 \mathrm{~dB}$ time of the power excursion at the output of the fifth EDFA stands at $7.4 \mu \mathrm{s}$, whereas the $1 \mathrm{~dB}$ time of the SNR excursion is $19.8 \mu \mathrm{s}$. Hence, the curves for the SNR cases are compressed, featuring width of $3.5 \mathrm{~dB}$ for the self-similar case 


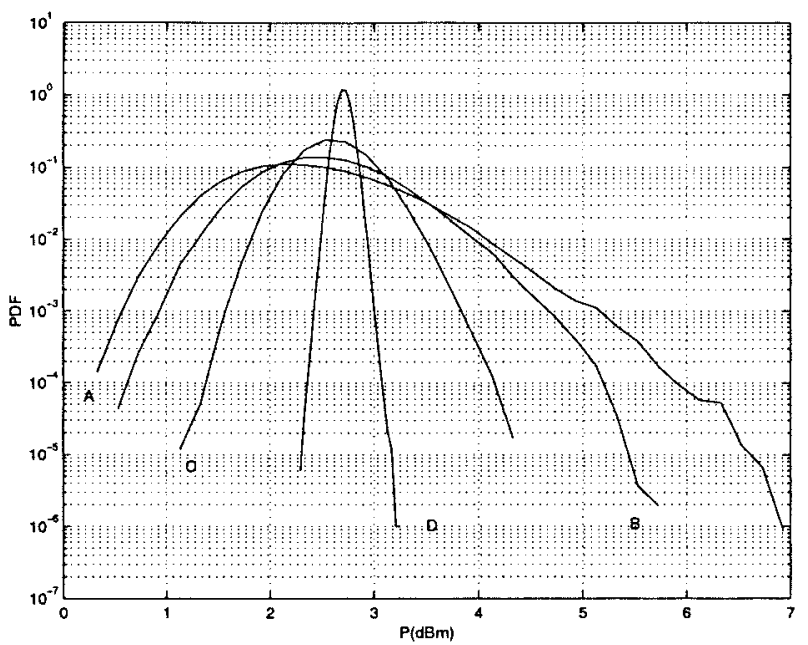

(a)

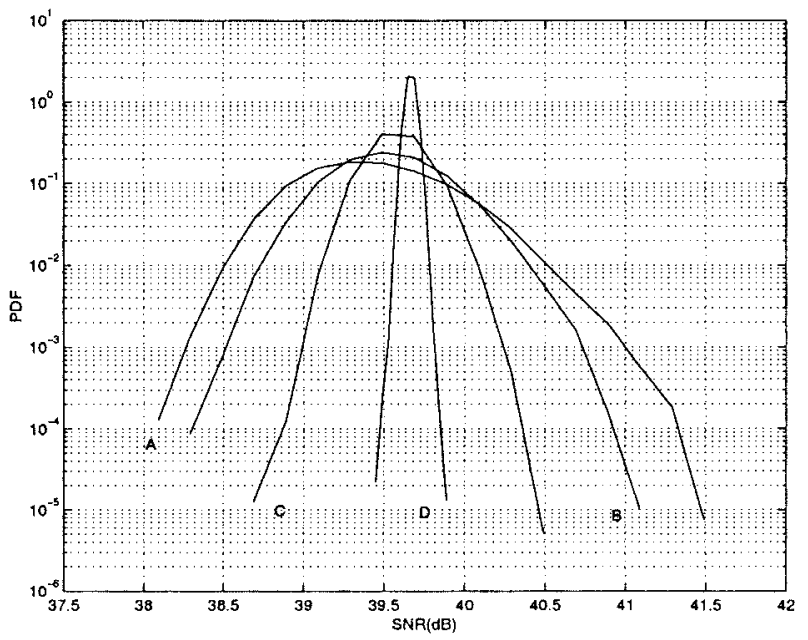

(c)

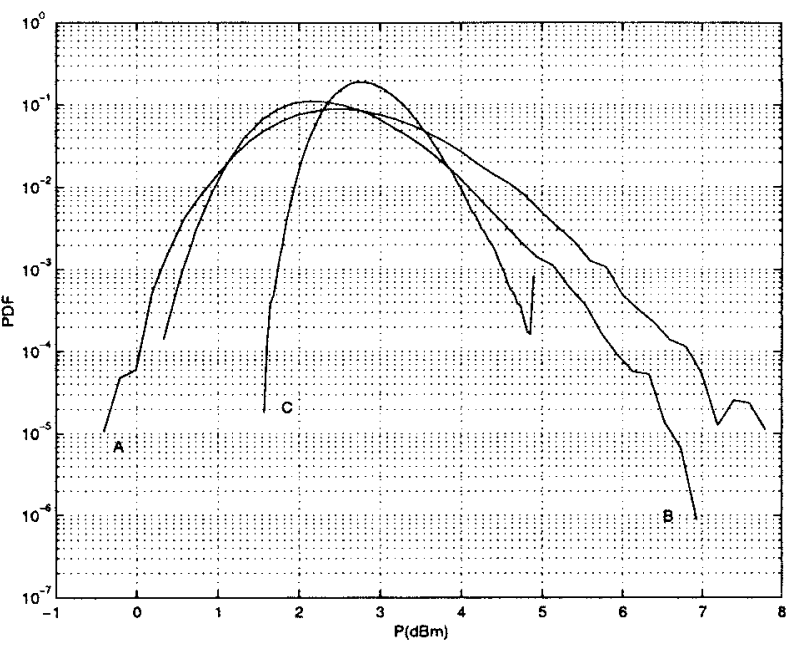

(b)

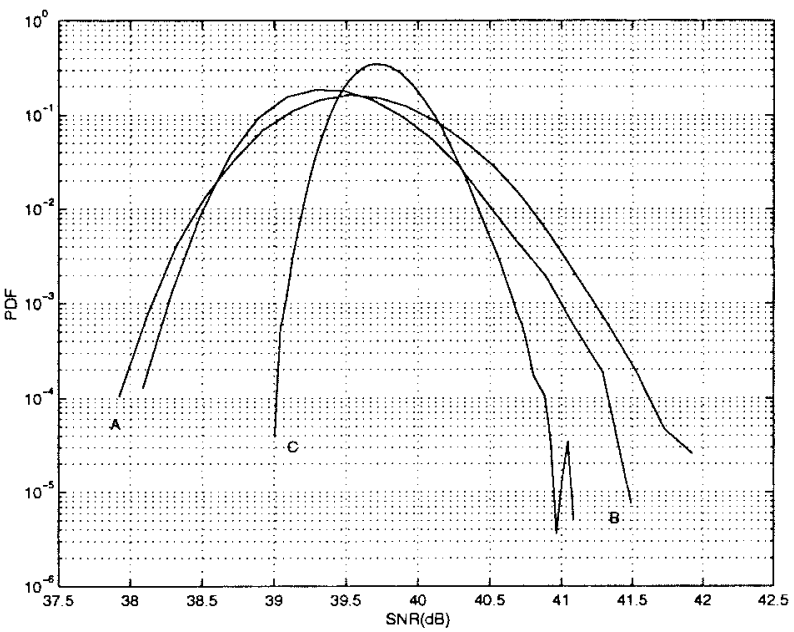

(d)

Fig. 7. Simulation of a cascade of EDFAs: (a) estimated probability density function of powers at the output of the 5th EDFA for utilization $\rho=0.5$ and various $\alpha$ parameters: $\alpha_{\text {off }}=\alpha_{\text {on }}=1.2$ (curve A); $\alpha_{\text {off }}=\alpha_{\text {on }}=1.2$ and $2.5 \mathrm{~Gb} / \mathrm{s}$ (curve B); $\alpha_{\text {off }}=\alpha_{\text {on }}=2.1$ (curve C); $\alpha_{\text {off }}=\alpha_{\text {on }}=5.0$ (curve D); (b) simulated probability density function of powers at the output of the fifth EDFA, self-similar case and various utilizations: $\alpha_{\text {on }}=1.64, \alpha_{\text {off }}=1.2$ $\rho=0.3$ (curve A); $\alpha_{\text {on }}=1.2, \alpha_{\text {off }}=1.2 \rho=0.5$ (curve B); $\alpha_{\text {on }}=1.2, \alpha_{\text {off }}=1.64 \rho=0.7$ (curve C); (c) simulated probability density function of SNR at the output of the fifth EDFA for utilization $\rho=0.5$ and various $\alpha$ parameters: $\alpha_{\text {off }}=\alpha_{\text {on }}=1.2$ (curve A); $\alpha_{\text {off }}=\alpha_{\text {on }}=1.2$ and $2.5 \mathrm{~Gb} / \mathrm{s}$ (curve B); $\alpha_{\text {off }}=\alpha_{\text {on }}=2.1$ (curve C); $\alpha_{\text {off }}=\alpha_{\text {on }}=5.0$ (curve D); (d) simulated probability density function of SNR at the output of the fifth EDFA, self-similar case and various utilizations: $\alpha_{\text {on }}=1.64, \alpha_{\text {off }}=1.2 \rho=0.3$ (curve A); $\alpha_{\text {on }}=1.2, \alpha_{\text {off }}=1.2 \rho=0.5$ (curve B); $\alpha_{\text {on }}=1.2, \alpha_{\text {off }}=1.64 \rho=0.7$ (curve C).

with $\rho=0.5$ and $\alpha_{\text {off }}=\alpha_{\text {on }}=1.2$ (curve A) as opposed to nearly $7 \mathrm{dBm}$ for the corresponding power histogram. Again the broadening is more pronounced as the utilization decreases and the variability increases. We note that the full load limits for the cases depicted in Fig. 7(d) are: $36.5 \mathrm{~dB}$ for $\rho=0.3$ (curve A); $38 \mathrm{~dB}$ for $\rho=0.5$ (curve B); and $39 \mathrm{~dB}$ for $\rho=0.7$ (curve C). Also note that curve $\mathrm{C}$ already reaches that asymptote. The main observation, however, is that the SNR swings are much smaller than the corresponding power swings. The explanation is that as the power increases/decreases due to the gain change in EDFA, so does the ASE noise, and hence the optical SNR, which is a ratio of these two quantities, assumes different values but at a much slower pace and at a much smaller size as compared to those in the power case. However, as indicated in Fig. 2(d), in long cascades of EDFA's this difference diminishes progressively and the SNR excursions become comparable to those of the power excursions.

\section{CONCLUSIONS}

We have presented a rather extensive set of simulations, in which we investigate the influence of the statistics of the input traffic on the statistics of the power and SNR at the output of an EDFA chain. By modeling the input traffic on each channel of a WDM system as a succession of ON-OFF periods, each with a Pareto heavy-tailed distribution, we have simulated input packetized traffic with various degrees of variability in the packet bursts. To capture the heavy tails of the ON-OFF distribution (important for traffic with large variability) we have monitored the transmission of one million slots, calculating the power transients in ten points per slot. The results of the simulations were used to build a histogram estimation of the probability density function of the output power and optical SNR, taken for input traffic with different variability and for different network utilization factors. The 
statistics were taken both at the output of the first EDFA in the cascade and at the output of the fifth EDFA in the cascade, giving ample opportunity to highlight the influence of the cascade. The results indicate that substantial power and SNR swings are present when an EDFA is used to amplify highly-variabile burst-mode packet traffic. The effect is much more pronounced along a cascade, where power swings in excess of $9 \mathrm{dBm}$ and SNR swings of more than $4 \mathrm{~dB}$ were observed during the course of one simulation with probability larger than $10^{-4}$. This can represent a serious problem, pointing at potential receiver dynamic range limitations, error bursts if the power exceeds certain thresholds for optical nonlinearities or inadequate eye opening. It should be stressed that this study determines this effect qualitatively in the case of highly variable multimedia traffic and secondly, it highlights that output power and SNR fluctuations are more acute in cascades of EDFA's. A quantitative analysis of the system impact of such power fluctuations depends very strongly on system parameters, as indicated by two parallel simulations for systems with significantly different EDFA parameters, leading to significantly different values for the amount of broadening of the power swing curves. We note in passing that recent developments make it possible to regenerate [21] and detect [22] high-speed data transmitted in burst packet mode. Finally, we note that the power and SNR swings will increase markedly when such traffic is fed into longer cascades of EDFA's, indicating a clear demand for a gain compensation mechanism.

\section{APPENDIX \\ $1 \mathrm{~dB}$ TIME}

The amplifier gain is a continuous function of time because of the existence of its time derivative in (1). However, a step transition in the input power leads to a step transition in the output power, and such discontinuity propagates along the chain of amplifiers. The amplifier gains evolve in time as per (1), and the system converges toward a new steady-state solution. The speed of the transients in the output power from one steady state solution to the next can be characterized by the time in which the output power changes by one $\mathrm{dB}$, i.e., the $1 \mathrm{~dB}$ time [2].

Let $P_{i, k}^{\text {in }}$ and $P_{i, k}^{\text {out }}$ be the input and output fluxes, respectively, of channel $k$ at amplifier $i$. Define the dBpower excursion of channel $k$ at the output of amplifier $i$ as $\Delta P_{i, k}^{d B}(t) \triangleq 10 \log _{10}\left[P_{i, k}^{\text {out }}(t) / P_{i, k}^{\text {out }}\left(0^{+}\right)\right]$where $t=0^{+}$ indicates one instant after the step transition at $t=0$. Approximating $\Delta P_{i, k}^{d B}(t)$ as a straight line in $t$ and defining the (normalized) slope

$$
\left.\Delta_{i, k} \triangleq \frac{d}{d t}\left(\ln P_{i, k}^{\text {out }}(t)\right)\right|_{t=0^{+}}
$$

we get a simple approximation of the $1 \mathrm{~dB}$ time as the inverse of the slope

$$
t_{1-\mathrm{dB}}=1 /\left(10 \log _{10} e \Delta_{i, k}\right)
$$

Given that the interamplifier losses are time invariant, the time derivative of the input power of one amplifier is equal to the time derivative of the output power of the previous amplifier, leading to the following recurrence:

$$
\begin{aligned}
\Delta_{i, k} & =\left.\frac{d}{d t}\left(\ln P_{i, k}^{\mathrm{in}}(t)\right)\right|_{t=0^{+}}+\left.\frac{d}{d t}\left(G_{i, k}(t)\right)\right|_{t=0^{+}} \\
& =\left.\frac{d}{d t}\left(\ln P_{i-1, k}^{\text {out }}(t)\right)\right|_{t=0^{+}}+\left.\frac{d}{d t}\left(G_{i, k}(t)\right)\right|_{t=0^{+}} \\
& =\Delta_{i-1, k}+\left.\frac{d}{d t}\left(G_{i, k}(t)\right)\right|_{t=0^{+}}
\end{aligned}
$$

where $\Delta_{0, k}=0$. This is a difference equation describing the slope evolution along the cascade with initial condition that the slope of the input power at the first amplifier is zero. Finally, assuming the system is at steady state before the transition, the derivative of the gain can be found by solving (1)

$$
\frac{d}{d t} G_{i, k}(t)=\sum_{j=0}^{N} \frac{\Delta P_{i, j}}{\tau P_{k}^{I S}}\left(e^{G_{i, j}(0)}-1\right)
$$

where $\Delta P_{i, j} \triangleq P_{i, j}^{\mathrm{in}}\left(0^{+}\right)-P_{i, j}^{\mathrm{in}}(0)$, and we have $\Delta P_{i, j}=$ $\Delta P_{i-1, j} e^{G_{i-1, j}} / L_{i-1, j}$, and $\Delta P_{0, j}$ is the step transition of the input power to the first amplifier. $L_{i-1, j}$ is the loss between amplifier $i-1$ and $j$.

The knowledge of the slope evolution along the cascade and the associated $1 \mathrm{~dB}$ time could be put into practical use in designing a gain control mechanism by switching the pump. In this scenario, the pump is switched after a certain amount of time has elapsed since the power transition. The amount of pump power change should be such that it provides an effective gain control-knowledge of the slope could be employed to design a pump compensation scheme that exactly counter-measures the slope by inducing an additional slope with the opposite sign. Hence, from (11), the pump change $\Delta P_{i, 0}$ (photons/s) necessary to compensate the slope at the $i$ th amplifier is given by

$$
\Delta P_{i, 0} \doteq \frac{1}{1-e^{G_{i, 0}(0)}} \sum_{j=0}^{N} \Delta P_{i, j}\left(e^{G_{i, j}(0)}-1\right)
$$

which is a photon flux conservation, valid also for steadystate compensation: the pump should increase (decrease) its flux so as to increase (decrease) the number of excited ions in the EDFA by the amount corresponding to the ions consumed/spared by the total channel flux variation. This expression is used in Section V to find the pump change necessary to keep the system well balanced on average for different utilization factors and to provide gain compensation when the network utilization changes.

\section{REFERENCES}

[1] Y. Sun, G. Luo, J. L. Zyskind, A. A. M. Saleh, A. K. Srivastava, and J. W. Sulhoff, "Model for gain dynamics in erbium-doped fiber amplifiers," Electron. Lett., vol. 32, no. 16, pp. 1490-1491, Aug. 1996.

[2] Y. Sun, A. K. Srivastava, J. L. Zyskind, J. W. Sulhoff, C. Wolf, and R. W. Tkach, "Fast power transients in WDM optical networks with cascaded EDFA's," Electron. Lett., vol. 33, no. 4, pp. 313-314, Feb. 1997.

[3] Y. Sun, A. A. M. Saleh, J. L. Zyskind, D. L. Wilson, A. K. Srivastava, and J. W. Sulhoff, "Time dependent perturbation theory and tones in cascaded Erbium-doped fiber amplifier systems," J. Lightwave Technol., vol. 15, pp. 1083-1087, July 1997. 
[4] E. Desurvire, "Analysis of transient gain saturation and recovery in Erbium-doped fiber amplifiers," IEEE Photon. Technol. Lett., vol. 1, pp. 196-199, Aug. 1990.

[5] C. R. Giles, E. Desurvire, and J. R. Simpson, "Transient gain and crosstalk in Erbium-doped fiber amplifiers," Opt. Lett., vol. 14, no. 16, pp. 880-882, Aug. 1989.

[6] A. Bononi and L. A. Rusch, "Doped-fiber amplifier dynamics: A system perspective," J. Lightwave Technol., vol. 16, pp. 945-956, May 1998.

[7] C. Qiao, "Optical buffer switching-A novel paradigm for gigabit WDM optical networks," in Proc. Gigabit Networking Symp., San Francisco, CA, Mar. 1998.

[8] W. E. Leland, M. S. Taqqu, W. Willinger, and D. V. Wilson, "On the self-similar nature of ethernet traffic (extended version)," IEEE/ACM Trans. Networking, vol. 2, pp. 1-15, Feb. 1994.

[9] J. Beran, R. Sherman, M. S. Taqqu, and W. Willinger, "Long-range dependence in variable-bit-rate video traffic," IEEE Trans. Commun., vol. 43, pp. 1566-1579, Feb./Mar./Apr. 1995.

[10] E. Desurvire, Erbium-Doped Fiber Amplifiers. New York: Wiley, 1994.

[11] T. Georges and E. Delevaque, "Analytic modeling of high-gain Erbiumdoped fiber amplifiers," Opt. Lett., vol. 17, no. 16, pp. 1113-1115, Aug 1992.

[12] A. Bononi, L. Barbieri, and L. A. Rusch, "Using SPICE to simulate gain dynamics in doped-fiber amplifier chains," presented at OFC '98, Workshop 204, Transmission modeling simulation tools, San Jose, CA, Feb. 23, 1998.

[13] Y. Sun, J. L. Zyskind, and A. K. Srivastava, "Average inversion level modeling, and physics of Erbium-doped fiber amplifiers," IEEE J. Select. Areas Quantum Electron., vol. 4, pp. 991-1007, Aug. 1997.

[14] W. Zeiler, F. Di Pasquale, P. Bayvel, and J. E. Midwinter, "Modeling of four-wave mixing and gain peaking in amplified WDM optical communication systems and networks," J. Lightwave Technol., vol. 14, pp. 1933-1942, Sept. 1996.

[15] A. E. Willner and S. Hwang, "Transmission of many WDM channels through a cascade of EDFA's in long-distance links and ring networks," J. Lightwave Technol., vol. 13, pp. 802-816, May 1995.

[16] M. A. Ali, A. F. Elrefaie, R. E. Wagner, and S. A. Ahmed, "A detailed comparison of the overall performance of 980 and $1480 \mathrm{~nm}$ pumped EDFA cascades in WDM multiple-access lightwave networks," J. Lightwave Technol., vol. 14, pp. 1436-1448, June 1996.

[17] M. S. Taqqu, W. Willinger, and R. Sherman, "Proof of a fundamental result in self-similar traffic modeling," Comput. Commun. Rev., vol. 27, no. 2, pp. 5-23, Apr. 1997.
[18] A. Erramilli, O. Narayan, and W. Willinger, "Experimental queueing analysis with long-range dependent packet traffic," IEEE/ACM Trans. Networking, vol. 4, pp. 209-223, Apr. 1996.

[19] M. W. Garrett and W. Willinger, "Analysis, modeling and generation of self-similar VBR video traffic," in Proc. SIGCOMM'94, pp. 269-280.

[20] A. Bononi, L. Taňcevski, and L. A. Rusch, "Fast dynamics and power swings in doped-fiber amplifiers fed by highly-variable multimedia traffic," in Proc. OFC'98, paper WM31.

[21] P. Gunning et al., "10-Gbit/s asynchronous digital optical regenerator," in Proc. OFC '99, paper TuJ5, San Diego, CA, 1999.

[22] Y. Yamada et al., "Ultrafast clock recovery for burst-mode optical packet communication," Proc. OFC '99, paper TuL7, San Diego, CA, 1999.

L. Tančevski, photograph and biography not available at the time of publication.

Alberto Bononi received the "Laurea in Ingegnería Elettronica" cum laude from the University of Pisa, Pisa, Italy, in 1988 and the M.A. and Ph.D. degrees in electrical engineering from Princeton University, Princeton, NJ, in 1992 and 1994, respectively.

From 1994 to 1996, he was an Assistant Professor in Electrical and Computer Engineering Department at the State University of New York at Buffalo (SUNY), Buffalo, NY, teaching courses on circuits and systems, and on optical networks. Since 1996, he has been an Assistant Professor at the University of Parma, Italy, where he teaches courses on communications networks, probability theory and stochastic processes. His main research interests include system design and performance issues in fast packet switching and high-speed all-optical networks, nonlinear fiber transmission design for WDM systems, and gain dynamics in fiber amplifier chains. He has been the scientific coordinator of the European Community sponsored project DAWRON on wavelength routed optical networks.

Leslie A. Rusch (S'91-M'94), for a photograph and biography, see p. 405 of the March 1999 issue of this JournAL. 